\title{
ACE-Inhibitor Related Angioedema Is Not Sufficiently Reported to the Danish Adverse Drug Reactions Database
}

\section{Johan Emil Lundbek}

Cornwall (D) ${ }^{\prime}$

Anette Bygum (iD ${ }^{1,2}$

Eva Rye Rasmussen $\mathbb{1}^{3}$

'Department of Dermatology and Allergy Centre, Odense University Hospital, Odense C 5000, Denmark; ${ }^{2}$ Department of Clinical Research, University of Southern Denmark, Odense, Denmark; ${ }^{3}$ Department of Otorhinolaryngology, Head \& Neck Surgery and Audiology, Rigshospitalet, University of Copenhagen, Copenhagen 2100, Denmark
Correspondence: Johan Emil Lundbek Cornwall

Department of Dermatology and Allergy Centre, Odense University Hospital, J.B.

Winsloewsvej 4, Entrance 142, Odense C 5000, Denmark

Tel +45 42172449

Email johan.cornwall@gmail.com
This article was published in the following Dove Press journal: Drug, Healthcare and Patient Safety

Purpose: The primary objective of this study was to calculate the report rate of angiotensinconverting enzyme inhibitor-related angioedema (ACEi-AE). Secondary objectives were to determine factors suspected to affect the likelihood of ACEi-AE being reported and to investigate potential differences in angioedema risks between different ACEis.

Patients and methods: Patient data from two cohorts comprising 176 patients with ACEiAE were compared with report data from the Danish Adverse Drug Reactions Database, administered by the Danish Medicines Agency (DKMA). The study period was 1994-2015. Data were linked using unique personal identification numbers and birth dates. Cohort data and report data were compared with ACEi sales numbers from MedStat, an official database containing annual pharmaceutical drug sale data in Denmark.

Results: ACEi-AE was reported in two out of 176 cases resulting in a report rate of $1.1 \%$, meaning that $98.9 \%$ of the cases were not reported. Since 1994, a total of 417 ACEi-AE reports were made to the DKMA. Fifty-eight percent of these were made by general practitioners or physicians with unknown workplaces and $35 \%$ by hospital staff. Enalapril and ramipril were the most sold ACEi's in the study period ( $40.3 \%$ and $42.6 \%$, respectively). Enalapril was associated with $54.7 \%$ of ACEi-AE reports while ramipril was associated with 14.2\%. ACEi substance received was known for 141 cohort patients, of which $53.9 \%$ were prescribed enalapril and $17.0 \%$ received ramipril.

Conclusion: ACEi-AE was found to be severely underreported in Denmark, greatly limiting the available incidence data for this potentially life-threatening adverse reaction.

Keywords: edema, side-effect, adverse-effect, pharmacovigilance, antihypertensive, agent

\section{Introduction}

Knowledge about and management of adverse drug reactions (ADRs) is an important part of modern medicine, as some can severely harm patients' health and potentially be life-threatening. ${ }^{1-3}$ One of the primary methods of pharmacovigilance is collecting data from spontaneous reports and storing these in ADR databases for analysis. ${ }^{2}$ These add to data from clinical studies, which often consist of selected study-populations and have limited follow-up periods. Also, adverse drug events in clinical trials are not always reported in optimal ways. ${ }^{4}$ A limitation of ADR databases is that they require a sufficient number of reports to avoid underestimating ADR incidences. Unfortunately, studies show a tendency for healthcare professionals to underreport severe and non-severe ADRs. ${ }^{5-8}$ 
The European Union (EU) implemented the pharmacovigilance legislation in 2012 due to increased awareness of ADRs causing $\sim 197,000$ deaths annually in the EU. ${ }^{9}$ The legislation was accompanied by an implementation regulation and a guideline on good pharmacovigilance practices. ${ }^{9}$ In Denmark (population of 5.7 million inhabitants), it is compulsory for physicians to report all ADRs for drugs marketed within 2 years, after which only unexpected and serious ADRs have to be reported. ${ }^{3,10}$ It is possible, but not required, for non-physician healthcare professionals, patients, relatives and other officials to report ADRs.

Angiotensin-Converting Enzyme-inhibitors (ACEi), which are commonly used to treat conditions such as hypertension, diabetic nephropathy and congestive heart failure, can sometimes cause severe swellings of skin and mucosa also known as angioedema (AE). ${ }^{11}$ ACEi-related $\mathrm{AE}$ (ACEi-AE) predominantly occurs in the head and neck area and has the potential to become life-threatening due to asphyxiation when located in the upper airways. ${ }^{12-15}$ It is important to terminate ACEi usage and register it as being contraindicated for ACEi-AE patients. ${ }^{12,16}$ Despite occurring in only $0.2-2.5 \%$ of the treated patients, ACEi$\mathrm{AE}$ has a relatively high prevalence, since more than 40 million people worldwide are currently receiving an ACEi and the usage is rising. ${ }^{16-18} \mathrm{AE}$ has been observed as an ADR of various other pharmaceuticals and in relation to other medical conditions, ie, allergy, making the diagnosis difficult. ${ }^{14,15,19,20}$ ACEi-AE can occur years after first drug dispensing, which may reduce suspicion of drug-related AE. ${ }^{14,21}$ No diagnostic test has been found to rapidly and effectively distinguish between AE subtypes. ${ }^{14,22}$ Failure to diagnose $\mathrm{ACEi}-\mathrm{AE}$ can be assumed to reduce the report rate and delay ACEi withdrawal, potentially endangering patients. ${ }^{14,15,22,23}$

\section{Aims}

The main objective of this study was to link two previously formed cohorts of Danish ACEi-AE patients with the Danish ADR Database to calculate the report rate. Secondary objectives were to determine factors related to the likelihood of an ADR being reported (ie, profession, department or severity of the ADR) and to identify potential differences in AE risks between ACEi's.

\section{Materials and Methods}

The STROBE guideline was employed during the execution of this study. ${ }^{24}$

\section{Study Design}

The present study is an observational retrospective cohort study. The study period was 1994-2015.

\section{Study Population}

The study population comprised 176 adult patients (71 males, 105 females) from two cohorts. Cohort 1 comprised 105 (38 males; 67 females) patients who were diagnosed with ACEi-AE. ${ }^{13}$ These patients were part of a larger AE cohort comprising 612 patients diagnosed at Odense University Hospital (DK) between 1994 and 2015 (unpublished data). The first ACEi-AE patient was diagnosed in 2003. Cohort 2 comprised 77 patients prospectively included in the PREDICTION-ADR project between 2014 and $2016 .{ }^{25}$ Six patients in cohort 2 were excluded since they were diagnosed with angiotensin II antagonistrelated $\mathrm{AE}$.

\section{The Danish ADR Database}

In Denmark, ADRs are reported electronically to the Danish Medicines Agency (DKMA). ${ }^{26}$ Data are stored in the Danish ADR Database. When reporting an ADR, it is mandatory to fill in symptoms, patient outcome, name of the suspected pharmaceutical and at least one identifier of the patient, which include birth date, age, personal identification number (the Danish Civil Registration System: CPR number), gender or patient initials. ${ }^{27}$

\section{Data Extraction from Danish ADR Database}

A request for a data export comprising all ACEi-AE reports was sent to the Danish ADR Database (see Supplementary Material 1). The linkage and further data retrieval were handled by a DKMA Special Advisor (pharmacist). Data import/export was handled through a secure hospital mailbox. Data from reports received from 1994 onwards were requested, as this was the earliest time that the study population could have been diagnosed with ACEi-AE. The CPR number was used to link each cohort patient data to the potential ADR report. Attempts (digital search in the database) were made to link the patient data to ADR reports using birth date for reports lacking CPR numbers. An overview of the study population and the ADR report data is shown in Figure 1.

The Danish ADR Database was searched for ACEi-AErelated terms using the international ADR coding system Medical Dictionary for Regulatory Activities (MedDRA), 


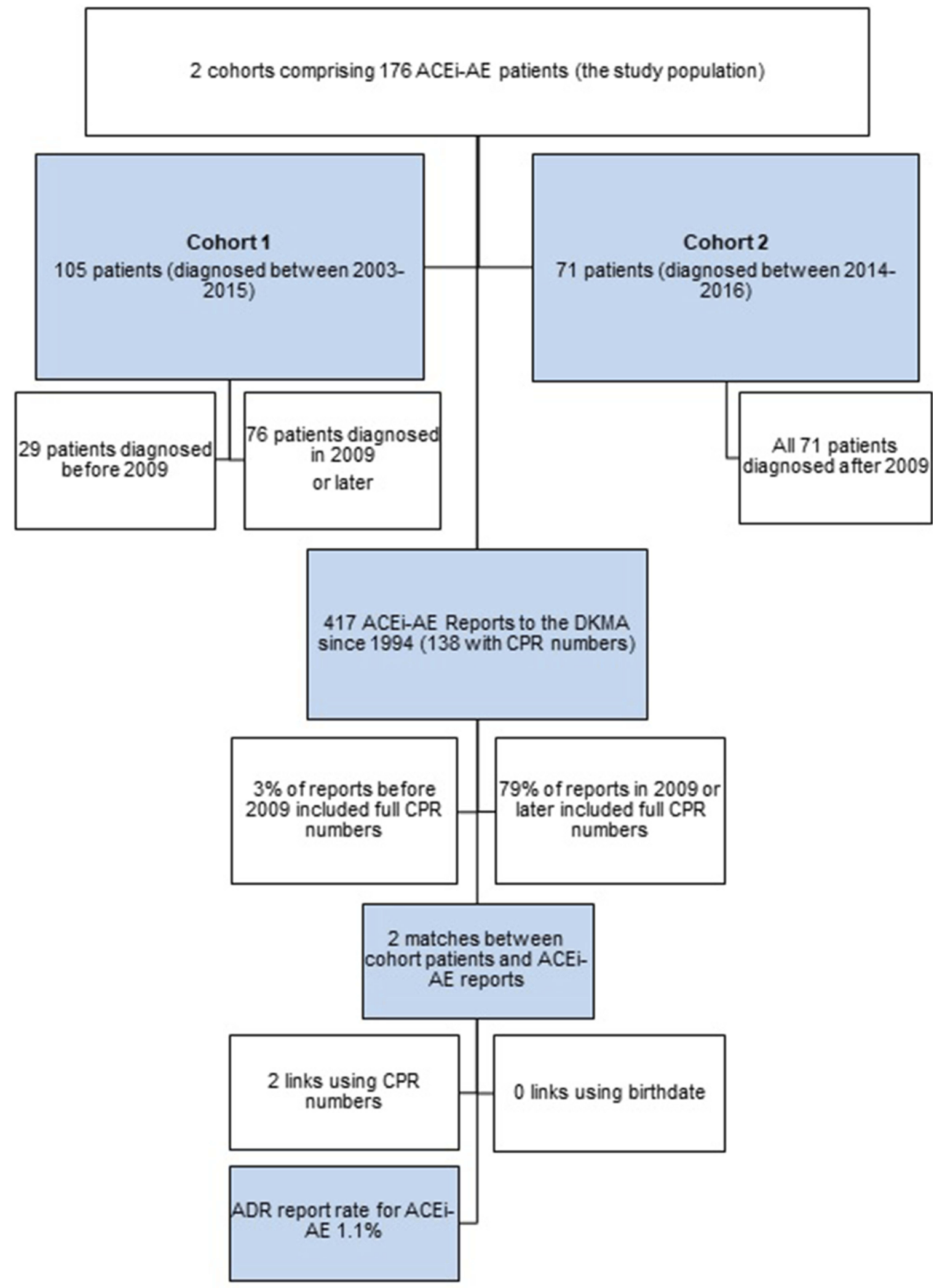

Figure I Structure of the present study.

Notes: Cohort $\mathrm{I}$ is a retrospective cohort study. ${ }^{13}$ Cohort 2 is a case-control study. ${ }^{25}$

Abbreviations: ACEi-AE, ACE-inhibitor-related angioedema; DKMA, Danish Medicines Agency; CPR, personal identification number. 


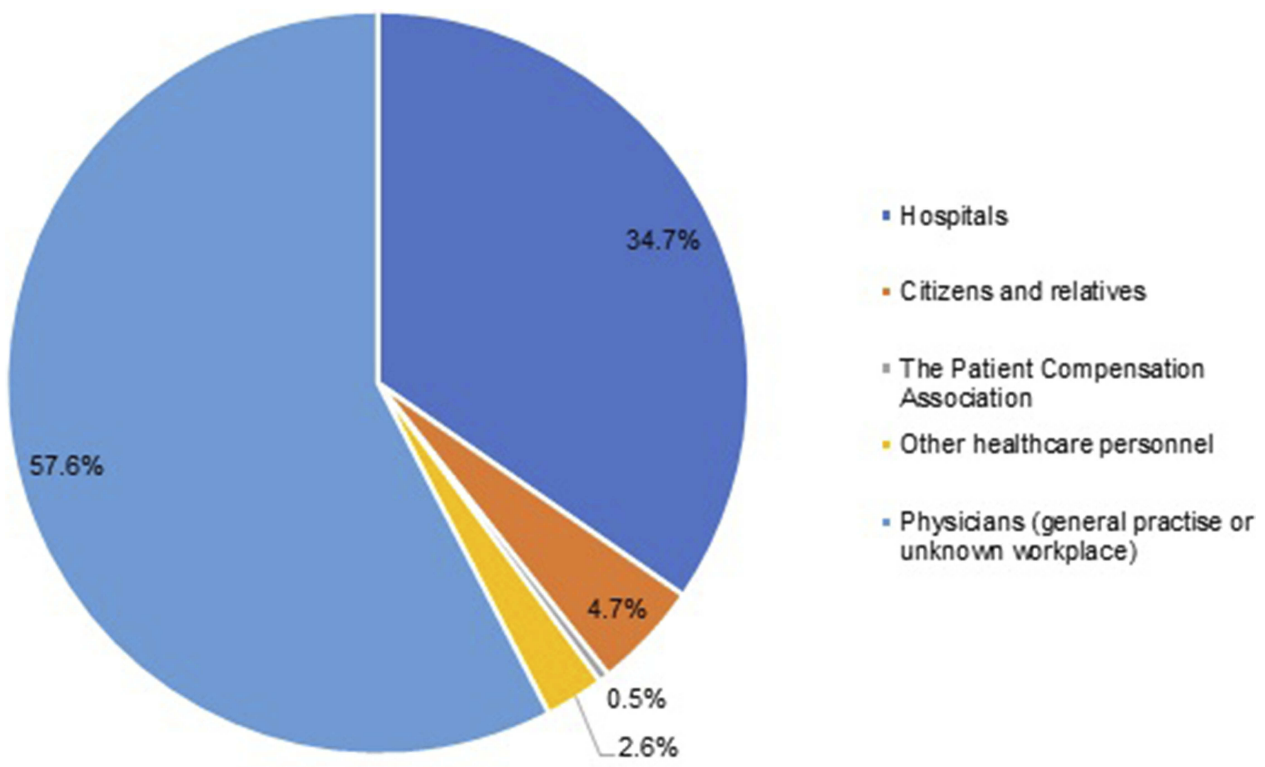

Figure 2 Distribution of origins of ACEi-AE reports to the DKMA (1994-2016). Abbreviations: ACEi-AE, ACE-inhibitor-related angioedema; DKMA, Danish Medicines Agency.

which contained 42 AE-related search terms (see Supplementary Material 2). The obtained information was used to calculate the proportion of ACEi-AE cases in the study population that were reported to the DKMA in order to estimate the report rate for ACEi-AE in Denmark in the study period.

\section{MedStat}

Data on medicine sales were collected from the MedStat Database, an official public database containing pharmaceutical drug sale data in Denmark between 1996 and 2017. ${ }^{28,29}$ An export of sales numbers for ACEi's sold in Denmark between 1997 and 2016 was created and the sales proportion for each ACEi substance was compared with the proportion of ACEi substances causing AE in the study population and causing reports to the DKMA, respectively.

\section{Statistical Methods}

Stata $^{\circledR}$ version 14 (StataCorp. 2015. Stata Statistical Software: Release 14. College Station, TX: StataCorp LP.) was used as a database system for the 105 ACEi-AE patients of cohort 1 and for statistical analysis. Study data on cohort 2 were collected and managed using REDCap (Research Electronic Data Capture) tools hosted at Odense University Hospital. ${ }^{30}$

\section{Ethical Considerations}

The Danish Data Protection Agency approved this study (case number 14/35206). The authors took every measure to ensure that no published results were personally identifiable.

\section{Results}

Linking the CPR numbers of the 176 patients from the study population to the ACEi-AE reports in the Danish ADR Database gave two matches. Attempting to link cohort patient data with the ADR reports that did not include CPR numbers using birth date did not provide additional matches. The calculated overall report rate for all studied ACEi-AE patients in the study population was two out of $176(1.1 \%)$. Of the two matches, one case (enalapril, 2010) was reported by a physician. The other (trandolapril, 2013) was reported by a lawyer.

According to the export from the Danish ADR Database, a total of 417 cases of ACEi-AE were reported since 1994; 138 included full CPR numbers. Only 3\% of ACEi-AE reports received before 2009 contained full CPR numbers, whereas that was the case for $79 \%$ of the reports received from 2009 onwards. The total number of annual ACEi-AE reports and the number of reports including CPR numbers can be seen in Supplementary Material 3. The patient data from cohort 1 (105 patients) were collected between 1994 and 2015 with the first ACEi-AE patient included in 2003. Twenty-nine patients were diagnosed between 2003 and 2008 while the remaining 76 were diagnosed in 2009 or later. The 71 patients from cohort 2 were diagnosed between 2014 and 2016 . 
$70.00 \%$

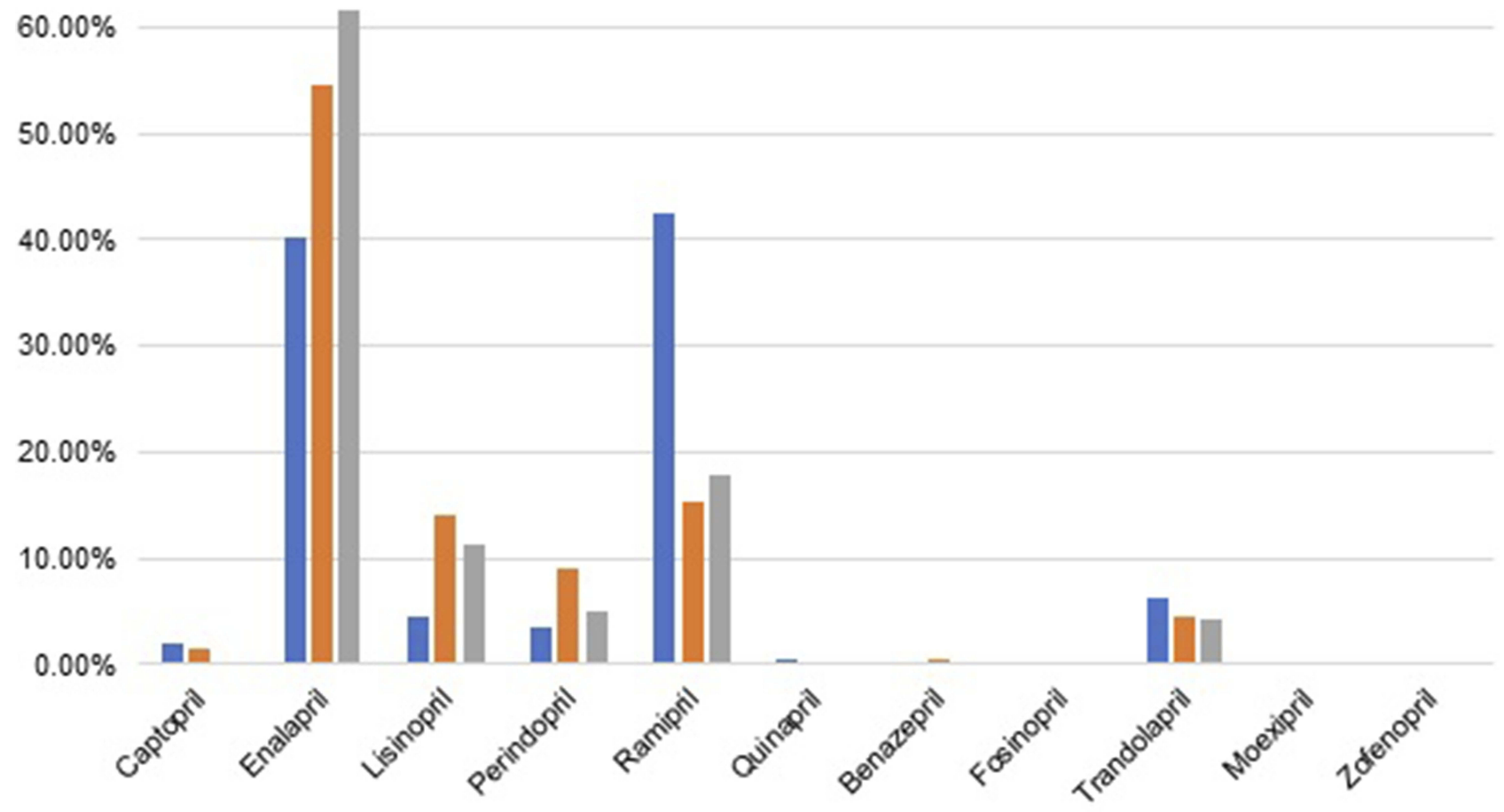

$\| \mathrm{ACE}$ i sale in Denmark $\quad \mathrm{ACE}$-AE reports to the DKMA $\| \mathrm{ACE}$ i recived by cohort patients

Figure 3 Distributions of ACEi substances received by cohort patients (1994-2015), ACEi substances related to ACEi-AE reports to the DKMA (I994-2016) and sales numbers of ACEi substances in Denmark (1997-2016).

Notes: Pharmaceutical drug sale data are from the MedStat database. ${ }^{29}$ Numbers represent calculated means for all years

Abbreviations: ACEi, ACE-inhibitor; ACEi-AE, ACE-inhibitor-related angioedema; DKMA, Danish Medicines Agency.

Fifty-eight percent of ACEi-AE reports were made by general practitioners or physicians with an unknown workplace (workplace not being compulsory to enter) and 35\% by hospital staff (primarily physicians). Reports by patients and relatives constituted $5 \%$ and other healthcare professionals reported 3\% (Figure 2).

ACEi-AE has been reported to the DKMA as an ADR of eight different ACEi's (Supplementary Materials 4 and 5). Enalapril usage was associated with $54.68 \%$ of reports, whereas ramipril was associated with $15.35 \%$ and lisinopril with $14.15 \%$. When searching the MedStat Database, it was found that eleven different ACEi-AE substances have been on the market between 1997 and 2016 in Denmark (Supplementary Materials 6 and 7 ). ${ }^{29}$ The majority of ACEi sales in this period consisted of enalapril (40.27\%) and ramipril (42.6\%). The active ACEi substances received by the study population were known in 141 of 176 cases. Seventy-six (53.9\%) of the 141 patients received enalapril and $24(17.0 \%)$ received ramipril (see Supplementary Materials 8). Over time the number of treated individuals in Denmark increased from 55.6 to 282 per 100.000 inhabitants (Supplementary Material 9). Enalapril was most frequently associated with AE in both the ADR database (54.7\%) and in the study population (54\%). Ramipril caused $15.35 \%$ of ACEi-AE reports and was received by $17 \%$ of the study population. A comparison of ACEi substances causing ADR reports, the drug-distribution in the study population and the Danish sales numbers can be found in Figure 3.

\section{Discussion}

The low ACEi-AE report rate found in this study (1.1\%) suggests that the actual incidence of ACEi-AE is much higher than what could be calculated using information from the Danish ADR Database. This lowers the reliability of the available ACEi-AE incidence data from the database and might lead to an overestimation of drug safety. There were an insufficient number of matches between cohort patient data and ACEi-AE reports to find any statistically significant correlations between variables 
mentioned in the "Aims" section of the introduction and likelihood of an ACEi-AE case being reported.

The primary method of data linkage was by comparing the CPR numbers, which presented a challenge, as the Danish ADR Database included reports without full CPR numbers. This was less of an issue when linking patient data to ADR reports made in 2009 or later, as only $21 \%$ of these lacked full CPR numbers. Since all data from cohort 2 were collected from patients diagnosed after 2009, the risk of not identifying patients from that cohort due to lack of CPR numbers in the database was low. This also applies to the data on the 76 patients in cohort 1 that were diagnosed in 2009 or later. Ninety-seven percent of ACEi-AE reports made before 2009 lacked CPR numbers, and thus the chance of a successful data linkage using only CPR was $3 \%$ for these reports. This limitation applies to 29 patients in the study population (29 in cohort 1 , none in cohort 2). To compensate for this, it was attempted to link the data of these 29 cohort patients to ACEi-AE reports by comparing their birth dates with the ones reported to the DKMA which did not result in additional matches.

As previously mentioned, $58 \%$ of ACEi-AE reports to the DKMA originated from general practitioners, while $35 \%$ came from hospital staff. Also, $47 \%$ of cohort 1 patients were referred from a general practitioner and the remaining 53\% from hospitals. ${ }^{13}$ The numbers from the Danish ADR Database suggest that Danish hospital staff is less likely to report ACEi-AE than general practitioners. However, data must be interpreted with caution, as the sample size is small. Also, it is possible that general practitioners review their patients more frequently than hospital physicians, potentially resulting in more opportunities to report ADRs. In a previous study by Rasmussen et al, it was found that $91.4 \%$ of ACEi-AE patients encountered either their general practitioner (44.76\%), the department of internal medicine (26.67\%), the emergency department $(10.48 \%)$ or the department of otolaryngology (9.52\%). A campaign aiming to increase ADR report rates for these medical specialties could, therefore, prove effective. $^{13}$

A Swedish study from 2004, which investigated reporting tendencies of different severe ADRs at five different Swedish hospitals, found an average report rate of $14 \%$ (15 reports of 92 cases). ${ }^{5}$ A systematic review conducted in the UK in 2009 found a median ADR underreporting rate of $94 \% .^{31}$ A retrospective study on the Thai ADR database investigated the characteristics of renin-angiotensin system-(RAS)-blocker-related AE and found only 895 relevant cases between 1984 and 2011 (37 years), despite Thailand having an estimated population of $68,414,135$ people. $^{32,33}$ ACEi-AE accounted for 785 (87.7\%) of these AE cases. Although the ADR report rates found in these studies were higher than in the present study, there seems to be a tendency to underreport ADRs.

In a systematic review conducted in 2009 by LopezGonzales et al, the most common determinants for underreporting of ADRs were identified to be: medical specialty, ignorance, diffidence, lethargy (including lack of interest or time to report), indifference, insecurity as to whether the drug is responsible for the ADR and complacency. ${ }^{34}$ This suggests that underreporting is linked to the attitudes that healthcare professionals have towards the importance of ADR reporting. ${ }^{34}$ Besides these determinants, ACEi-AE is, as previously mentioned, commonly misdiagnosed as allergic reactions, which could cause physicians to doubt the association between the patients' AE and ACEi usage, possibly accounting for the relatively lower ADR report rate in the present study compared to other studies.

The main alternative to collecting and analyzing ADR data from spontaneous reports is to obtain ADR incidence data from clinical studies, although this may not be optimal, especially for ADRs like ACEi-AE. Firstly, ACEi-AE is a rare condition per treatment $(0.2-2.5 \%)$, meaning that very large study populations could be needed to obtain statistically significant data. Secondly, ACEi-AE does not necessarily occur close to the start of treatment, resulting in a need for study periods lasting several years. ${ }^{35}$ Thirdly, clinical studies have selected study populations, which increases the risk of various biases and confounders, as opposed to ADR databases, that are designed to include data from all patients receiving the medication.

A randomized controlled trial, where physicians received educational intervention to improve ADR report rates, was conducted in $2014 .{ }^{36}$ A relative increase of $65.4 \%$ in ADR report rates was found after 8 months of follow-up, which suggests that education might improve reporting tendencies. However, if the report rate found in the present study $(1.1 \%)$ is taken into consideration, a relative increase of $65.4 \%$ is unlikely to substantially improve the quality of ACEi-AE data in ADR databases. Also, the results of ADR education seem to quickly fade, possibly making frequent sessions necessary to secure long-term effects on ADR report rates. ${ }^{36}$

In Denmark, a new telephone hotline was established in 2013, where healthcare professionals are able to request suspected ADRs to be reported by a professional. This 
significantly increased spontaneous ADR report rates during a pilot-study period. ${ }^{37}$

When comparing the ACEi substances in ACEi-AE reports to the Danish ADR Database with ACEi sales numbers, enalapril usage was found to be more associated with ACEi-AE than ramipril. This must be interpreted with caution, as the probability of a given active ACEi substance being reported could be subject to confounding (ie, local practices). However, the same tendency was found in the study population, suggesting that enalapril might render treated patients more susceptible to ACEiAE. A plausible explanation could be the long plasma half-life of enalapril (30 hrs) compared to ramipril (12$15 \mathrm{hrs}$ ). Also, ACEi-induced cough seems to be associated with long-acting ACEi's. ${ }^{38}$ Other studies have found a higher incidence of ACEi-AE in patients treated with enalapril compared to other ACEis. ${ }^{39}$

\section{Conclusion}

This study has found ACEi-AE to be severely underreported in Denmark. Only $1.1 \%$ of ACEi-AE cases in our validated study populations were reported to the DKMA, which limits data quality with regards to prevalence and associated factors.

\section{Acknowledgments}

The adverse drug reaction reports and related figures and tables referred to in this article originate from the Danish ADR Database administered by the DKMA. The cases have been forwarded to all relevant pharmaceutical companies and to the EudraVigilance Database. The pharmaceutical companies are not to report these cases to the DKMA. The authors thank Shire and CLS Behring for their contribution to AE research. Data regarding cohort 2 were stored by OPEN, Open Patient data Explorative Network, Odense University Hospital, Odense, Denmark (www.sdu.dk/ki/open). Study data were collected and managed using REDCap electronic data capture tools hosted at Odense University Hospital. ${ }^{30}$ We thank DKMA Special Advisor Mai Frederiksen Raun, who was responsible for the data export from the Danish ADR Database. Her constructive comments and feedback on the manuscript are greatly appreciated and contribute to the quality of this article.

\section{Author Contributions}

All authors contributed to data analysis, drafting or revising the article, gave final approval of the version to be published, and agree to be accountable for all aspects of the work. All authors fulfilled the authorship criteria in the IMCJE guidelines and have approved the final version of the manuscript. JC has written the manuscript, performed literature research and produced figures and tables. AB has provided data, performed data linkage, performed literature research and critically revised the manuscript, figures and tables. ER has conceived the idea for the study, provided data, performed data linkage, produced figures, performed literature research and critically revised the manuscript, figures and tables.

\section{Disclosure}

Johan Cornwall declares that he has no conflicts of interest. Dr. Eva Rye Rasmussen has previously performed scientific work in cooperation with Shire/Takeda. She has also received personal fees for educational seminars from MSD Norway and Shire/Takeda. CLS Behring and Viropharma have previously supported AE research performed by Dr. Eva Rye Rasmussen. Anette Bygum has received research grant support and/or speaker/consultancy fees from CSL Behring, Viropharma, SOBI and Shire/Takeda and participated in clinical trials for Jerini AG, Shire/Takeda and BioCryst. She is an advisor for the HAE Scandinavian Patient Organization. She participates in a clinical study in collaboration with BioCryst. Viropharma is now part of Shire/Takeda.

\section{References}

1. Lazarou J, Pomeranz BH, Corey PN. Incidence of adverse drug reactions in hospitalized patients: a meta-analysis of prospective studies. JAMA. 1998;279(15):1200-1205. doi:10.1001/jama.279.15.1200

2. Wester K, Jonsson A, Spigset O, Hagg S. Spontaneously reported fatal suspected adverse drug reactions: a 10-year survey from Sweden. Pharmacoepidemiol Drug Saf. 2007;16(2):173-180. doi:10.1002/ pds. 1263

3. Bangsgaard N, Saunte DM, Folkenberg M, Zachariae C. Serious adverse events reporting on systemic terbinafine: a Danish registerbased study. Acta Derm Venereol. 2011;91(3):358-359. doi:10.2340/ 00015555-1041

4. Seruga B, Templeton AJ, Badillo FEV, Ocana A, Amir E, Tannock IF. Under-reporting of harm in clinical trials. Lancet Oncol. 2016;17(5): e209-e219. doi:10.1016/S1470-2045(16)00152-2

5. Backstrom M, Mjorndal T, Dahlqvist R. Under-reporting of serious adverse drug reactions in Sweden. Pharmacoepidemiol Drug Saf. 2004;13(7):483-487. doi:10.1002/pds.962

6. Belton KJ. Attitude survey of adverse drug-reaction reporting by health care professionals across the European Union. The European Pharmacovigilance Research Group. Eur J Clin Pharmacol. 1997;52 (6):423-427. doi:10.1007/s002280050314

7. Hasford J, Goettler M, Munter K-H, Muller-Oerlinghausen B. Physicians' knowledge and attitudes regarding the spontaneous reporting system for adverse drug reactions. J Clin Epidemiol. 2002;55 (9):945-950. doi:10.1016/S0895-4356(02)00450-X 
8. Gahr M, Eller J, Connemann BJ, Schonfeldt-Lecuona C. Subjective reasons for non-reporting of adverse drug reactions in a sample of physicians in outpatient care. Pharmacopsychiatry. 2016;49(2):5761. doi:10.1055/s-0035-1569291

9. Hidalgo-Simon A, Arlett P. The pharmacovigilance legislation. Eur Med Agency. 2012;5(5):485-488. doi:10.1586/ecp.12.46

10. The Danish Health Authority. Bekendtgørelse om indberetning af bivirkninger ved lægemidler m.m; 2014. Available from: https:// www.retsinformation.dk/Forms/R0710.aspx?id=162794. Accessed August 2, 2017.

11. National Institutes of Health. DailyMed; 2017. Available from: https://dailymed.nlm.nih.gov/dailymed/. Accessed July 28, 2017.

12. Holm JPY, Ovesen T. Increasing rate of angiotensin-converting enzyme inhibitor-related upper airway angio-oedema. Dan Med J. 2009;59(6):A4449.

13. Rasmussen ER, Buchwald V, Wadelius M, et al. Assessment of 105 patients with angiotensin converting enzyme-inhibitor induced angioedema. Int $J$ Otolaryngol. 2017;2017:1-7. doi:10.1155/2017/ 1476402 .

14. Agostoni A, Cicardi M. Drug-induced angioedema without urticaria. Drug Saf. 2001;24(8):599-606. doi:10.2165/00002018-20012408000004

15. Jaiganesh T, Wiese M, Hollingsworth J, et al. Acute angioedema: recognition and management in the emergency department. Eur $J$ Emerg Med. 2013;20(1):10-17. doi:10.1097/MEJ.0b013e328356f76e

16. Rasmussen ER, Mey K, Bygum A. Angiotensin-converting enzyme inhibitor-induced angioedema-a dangerous new epidemic. Acta Derm Venereol. 2014;94(3):260-264. doi:10.2340/00015555-1760

17. Sánchez-Borges M, González-Aveledo LA. Angiotensin-converting enzyme inhibitors and angioedema. Allergy Asthma Immunol Res. 2010;2(3):195-198. doi:10.4168/aair.2010.2.3.195

18. Holm JPY, Ovesen T. Increasing rate of angiotensin-converting enzyme inhibitor-related upper airway angio-oedema. Dan Med J. 2012;59(6):A4449.

19. Tai S, Mascaro M, Goldstein NA. Angioedema: a review of 367 episodes presenting to three tertiary care hospitals. Ann Otol Rhinol Laryngol. 2010;119(12):836-841. doi:10.1177/000348941011901208

20. Scott SI, Andersen MF, Aagaard L, Buchwald CV, Rasmussen ER. Dipeptidyl peptidase-4 inhibitor induced angioedema - an overlooked adverse drug reaction? Curr Diabetes Rev. 2017. doi:10.2174/15733998136661702141138

21. Agostoni A, Cicardi M, Cugno M, Zingale LC, Gioffré D, Nussberger J. Angioedema due to angiotensin-converting enzyme inhibitors. Immunopharmacology. 1999;44(1):21-25. doi:10.1016/ S0162-3109(99)00107-1

22. Bernstein JA, Cremonesi P, Hoffmann TK, Hollingsworth J. Angioedema in the emergency department: a practical guide to differential diagnosis and management. Int J Emerg Med. 2017;10 (1):15. doi:10.1186/s12245-017-0141-z

23. Stone CJ, Brown NJ. Angiotensin-converting enzyme inhibitor and other drug-associated angioedema. Immunol Allergy Clin North Am. 2017;37(3):483-495. doi:10.1016/j.iac.2017.04.006

24. Initiative S. STROBE statement-checklist of items that should be included in reports of cohort studies. Available from: https://www. strobe-statement.org/fileadmin/Strobe/uploads/checklists/STROBE checklist_v4_cohort.pdf. Accessed May 2, 2018.
25. University of Dundee. Prediction-ADR; 2017. Available from: https://sites.dundee.ac.uk/prediction-adr/. Accessed December 10, 2019.

26. Danish Medicines Agency. Report side effects in humans from medicines and vaccines; 2017. Available from: https:/laegemiddelstyrelsen. $\mathrm{dk} / \mathrm{en} /$ sideeffects/side-effects-from-medicines/report-a-side-effect/ / link.aspx?_id=6B84AE2577A04EB29B0A47F444BD6548\&_z=z. Accessed October 24, 2017.

27. Pedersen CB. The Danish civil registration system. Scand J Public Health. 2011;39(7 Suppl):22-25. doi:10.1177/1403494810387965

28. Schmidt M, Hallas J, Laursen M, Friis S. Data resource profile: Danish online drug use statistics (MEDSTAT). Int J Epidemiol. 2016;45(5):1401-1402g. doi:10.1093/ije/dyw116

29. The Danish Health Data Authority. Statistics on the total sales of medicines in Denmark 1996-2015; 2016. Available from: http://med stat.dk/en. Accessed December 30, 2017.

30. Harris PA, Taylor R, Thielke R, Payne J, Gonzalez N, Conde JG. Research electronic data capture (REDCap)-a metadata-driven methodology and workflow process for providing translational research informatics support. J Biomed Inf. 2009;42(2):377-381. doi:10.1016/ j.jbi.2008.08.010

31. Hazell L, Shakir SAW. Under-reporting of adverse drug reactions: a systematic review. Drug Saf. 2006;29(5):385-396. doi:10.2165/ 00002018-200629050-00003

32. Central Intelligence Agency. East \& Southeast Asia: Thailand; 2017. Available from: https://www.cia.gov/library/publications/the-worldfactbook/geos/th.html. Accessed December 10, 2017.

33. Win TSZ, Chaiyakunapruk N, Suwankesawong W, Dilokthornsakul P, Nathisuwan S. Renin angiotensin system blockers-associated angioedema in the Thai population: analysis from Thai National Pharmacovigilance Database. Asian Pac J Allergy Immunol. 2015;33(3):227-235. doi:10.12932/AP0556.33.3.2015

34. Lopez-Gonzalez E, Herdeiro MT, Figueiras A. Determinants of under-reporting of adverse drug reactions: a systematic review. Drug Saf. 2009;32(1):19-31. doi:10.2165/00002018-20093201000002

35. Wakefield YS, Theaker ED, Pemberton MN. Angiotensin converting enzyme inhibitors and delayed onset, recurrent angioedema of the head and neck. Br Dent J. 2008;205(10):553-556. doi:10.1038/sj. bdj.2008.982

36. Lopez-Gonzalez E, Herdeiro MT, Pineiro-Lamas M, Figueiras A. Effect of an educational intervention to improve adverse drug reaction reporting in physicians: a cluster randomized controlled trial. Drug Saf. 2014;38(2):189-196. doi:10.1007/s40264-0140256-6

37. Vinther S, Klarskov P, Borgeskov H, et al. An adverse drug event manager facilitates spontaneous reporting of adverse drug reactions. Dan Med J. 2017;64(1).

38. Town GI, Hallwright GP, Maling TJ, O’Donnell TV. Angiotensin converting enzyme inhibitors and cough. $N$ Z Med J. 1987;100 (820):161-163.

39. Montoro de Francisco A, Garcia-Luque A, Fernandez M, Puerro M. Side effects of angiotensin converting enzyme inhibitors and angiotensin II receptor antagonists: are we facing a new syndrome. Am J Cardiol. 2012;110(10):1552-1553. doi:10.1016/j.amjcard.2012.08.027 


\section{Publish your work in this journal}

Drug, Healthcare and Patient Safety is an international, peer-reviewed open-access journal exploring patient safety issues in the healthcare continuum from diagnostic and screening interventions through to treatment, drug therapy and surgery. The journal is characterized by the rapid reporting of reviews, original research, clinical, epidemiological and post-marketing surveillance studies, risk management, health

literacy and educational programs across all areas of healthcare delivery. The manuscript management system is completely online and includes a very quick and fair peer-review system. Visit http://www.dovepress.com/testimonials.php to read real quotes from published authors.

Submit your manuscript here: https://www.dovepress.com/drug-healthcare-and-patient-safety-journal 